\title{
Surgical Interbody Research Group-radiographic assessment of interbody fusion devices: fusion criteria for anterior lumbar interbody surgery
}

\author{
J. Kenneth Burkus, M.D., Kevin Foley, M.D., Regis HaID, M.D., AND \\ Jean-Charles LeHuec, M.D., Ph.D.
}

The Hughston Clinic, Columbus, Georgia; Memphis, Tennessee; Department of Neurosurgery, Emory University, Atlanta, Georgia; and University of Bordeaux, Bordeaux, France

\begin{abstract}
The authors present their radiographic criteria for assessing fusion of the lumbar spine after anterior interbody fusion with intradiscal implants. These criteria include the assessment of plain radiographs, dynamic motion radiographs, and thin-cut computerized tomography scans. Fusion within the instrumented spinal motion segment can be determined using radiographic evaluation to assess spinal alignment on sequential examinations, angular and translational changes on dynamic motion studies, and device-host interface, and to identify new bone formation and bone remodeling. Finally, to aid the clinician in assessing fusion, the authors describe the five zones of fusion within the intervertebral disc space.
\end{abstract}

\author{
KEY WORDS • degenerative disc disease • interbody fusion device • radiography • \\ lumbar spine $\quad$ spinal fusion
}

One of the goals of ALIF is to create a solid arthrodesis across a spinal motion segment. A wide range of fusion rates for the procedure has been reported (unpublished data, Burkus and associates). ${ }^{2,6,9,12,13,16,18,20,22}$ The variability in fusion rates is, in part, determined by patient selection and surgical technique and also by the investigator's definition of fusion. No uniform methods or criteria for determining fusion after lumbar interbody fusion have been established. ${ }^{7,24}$

Interbody fusion is assessed by various imaging modalities; however, no single study or technique has been identified as being definitive in establishing the presence of fusion or pseudarthrosis after surgery. ${ }^{23}$ The most commonly used methods of assessing fusion are the interpretation of plain radiographs, dynamic radiographs, Tc bone scans, biplanar tomographs, axial CT scans, and MR images.

The use of interbody fusion devices creates new challenges in establishing fusion criteria, as these devices often obscure vertebral landmarks used in the assessment of fusion and can create artifacts and degrade the quality of some imaging modalities. ${ }^{1}$ Direct surgical exploration is not a viable option for determining the status of an interbody fusion. ${ }^{10}$ Indirect assessment of an anterior fusion through the intraoperative manipulation of the posterior

Abbreviations used in this paper: $\mathrm{ALIF}=$ anterior lumbar interbody fusion; $\mathrm{AP}=$ anteroposterior; $\mathrm{CT}=$ computerized tomography; MR = magnetic resonance. spinous processes can identify only gross patterns of instability and cannot reliably identify micromotion across an interspace.

\section{IMAGING STUDIES}

\section{Plain Radiography}

Plain radiographic studies used most often to assess fusion are obtained in the following patient positions: 1) standing or weight-bearing; 2) supine; 3) dynamic flexion-extension; and 4) dynamic side-bending. Standing or weight-bearing $\mathrm{x}$-ray films are considered more valuable than those obtained in the supine position. Using a weightbearing radiographic technique with stress on the interbody fusion can help identify sagittal- or frontal-plane instability patterns. The following criteria indicate fusion on plain radiographic studies: 1) incorporation of grafts to vertebral endplates; 2) bridging trabecular bone across the interspace; 3) absence of lucencies at the graft-host interface; 4) absence of subsidence; and 5) absence of graft migration.

Threaded cylindrical implants create artifacts that make interpretation of plain radiographs alone inaccurate in the determination of fusion. ${ }^{1}$ Bone growth within the implants cannot be assessed accurately on plain radiographs. The thread patterns of cylindrical devices create varying amounts of artifact at the implant-host bone interface. Radiographic lucencies in this area can be misinterpreted. 


\section{Dynamic Plain Radiography}

Dynamic motion studies of the lumbar spine are conducted in an attempt to identify subtle changes of the spinal motion segment. Criteria for fusion on dynamic radiographic studies are as follows: 1) angular motion of less than 3 to $5^{\circ}$; and 2 ) reduction in sagittal- or frontalplane translation of less than $5 \mathrm{~mm}$. The documentation of persistent motion across a fused motion segment has led some clinicians and researchers to conclude that the error in measuring dynamic plain radiographs often precludes an accurate determination of fusion. Biplanar radiographic techniques have been introduced to reduce measurement error. ${ }^{15}$

\section{Tomographic Studies}

Biplanar tomography and axial CT scanning have also been used to establish the success or failure of fusion. ${ }^{17,19}$ These studies reveal trabecular bone formation patterns within the intervertebral disc space. They are used to identify bridging bone formation that crosses the interspace. The studies can also identify lucencies at the implantbone interface. In our clinical experience, thin-cut CT scanning is the most precise and accurate technique for evaluating interbody fusion.

\section{Technetium Bone Scanning and MR Imaging}

Technetium bone scanning identifies regions of intense osteoblastic activity. This imaging modality has been used to assess spinal fusions; ${ }^{21}$ however, clinical studies have not shown this test to be reliable in detecting pseudarthrosis that is otherwise documented surgically. ${ }^{8}$

Magnetic resonance imaging has also been used to evaluate spinal fusions. The MR images can be used to identify persistent inflammatory changes within a spinal motion segment after surgery. ${ }^{4}$ Low-intensity signal on $\mathrm{T}_{1}$ weighted MR images represents micromotion at a fused interspace. These studies can be an efficacious method of evaluating allografts and carbon fiber implants; however, they are ineffective when titanium implants are present.

\section{FUSION ASSESSMENT}

Bone formation within a first-generation interbody fusion device is difficult to confirm. In the initial clinical studies involving the BAK cage (Sulzer Spine-Tech, Minneapolis, MN), CT scanning was not used to evaluate interbody fusion. Recently, Heithoff and coworkers proposed that the CT scan is of value in determining fusion in cases in which a BAK cage has been used (unpublished data). McAfee ${ }^{14}$ has advocated "ream long, fuse short" and packing bone graft anterior to BAK cages to achieve fusion. With this technique, the cages are placed in an area of the endplate that is least capable of supporting the stand-alone devices (unpublished data, Grant). Performance of this technique resulted in subsidence, loss of lordosis, delayed bone union, and pseudarthrosis within the instrumented spinal motion segment (Fig. 1). ${ }^{5}$ Furthermore, the "sentinel sign" of the progressive anterior bone formation frequently represented radial bone spur formation, not interbody fusion. The isolated sentinel sign may indicate progressive instability rather than progressive fusion. A new criterion should be established in which stan-

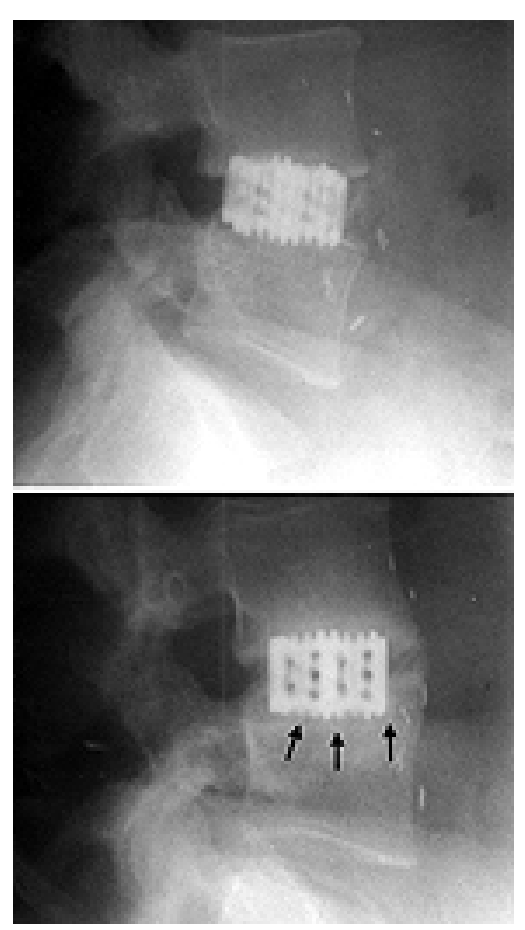

Fig. 1. Upper: Postoperative lateral radiograph revealing two BAK cages within the L4-5 interspace. The cages are recessed behind the anterior wall of the vertebral bodies and away from the ring apophysis anteriorly. Morselized bone graft has been placed in front of the cages. The cages are seated on top of the vertebral endplates. Lower: Lateral radiograph obtained 1 year postoperatively demonstrating significant subsidence of the BAK implants into the L-5 vertebral body. The arrows outline the extent of subsidence within the central portion of the L-5 vertebral body. A loss of segmental lordosis can be seen in comparison with the initial postoperative radiograph. There is an obvious pseudarthrosis despite the presence of an isolated sentinel sign.

dard radiographic techniques are used. More importantly, the need to assess fusion accurately should not prompt the surgeon to compromise his or her surgical technique.

The assessment of fusion in the presence of an interbody fusion device must include the radiographic evaluation of four key elements: 1) spinal alignment; 2) dynamic motion studies; 3) device-host bone interface; and 4) new bone formation and bone remodeling. Spinal alignment must be maintained over time. With a fused motion segment, the sagittal- and frontal-plane contours should not change. Similarly, with an intact fusion, no significant angular or translational change should be observed on dynamic motion studies. To determine intervertebral fusion in a patient in whom an interbody fusion device has been implanted, the contact points between the device and the host cortical bone and cancellous bone must be assessed. Identification of new trabecular bone formation within the disc space as well as remodeling of the grafts within the interbody devices and around the devices must also be assessed.

No single radiographic modality can be used to determine fusion accurately. Plain radiographs are effective in determining changes in spinal alignment over time. Dynamic plain radiographs can accurately assess changes in 


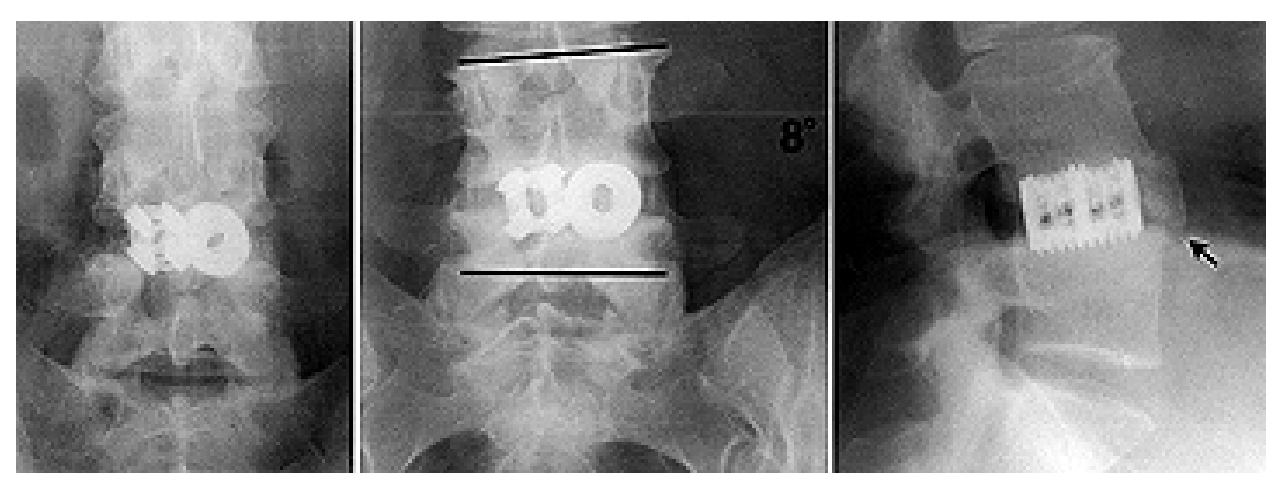

Fig. 2. Left: Postoperative AP radiograph showing a BAK cage and the BAK/Proximity device (Sulzer Spine-Tech) placed in the L4-5 interspace. There is no frontal-plane angulation at the L4-5 interspace. Center: Anteroposterior radiograph obtained 1 year postoperatively demonstrating rotation and asymmetrical subsidence of the BAK/Proximity implant into the L-5 vertebral body. An angular scoliotic deformity is now present at the L4-5 interspace. Right: Lateral radiograph showing that the implants were recessed away from the anterior ring apophysis and that bone was placed anteriorly. The sentinel sign is present; however, the graft has not attached to the L-5 vertebral body. The arrow points to the pseudarthrosis. Inadequate incorporation of the sentinel graft has permitted subsidence and angular deformity at the interspace.

implant-host bone interface and instability patterns within the spinal motion segment. Computerized tomography studies can identify new bone formation and bone remodeling within and around the spinal implants. By using all three imaging technologies, the physician can reliably determine the status of interbody fusion.

\section{Spinal Alignment}

The use of stand-alone implants in ALIF often improves the frontal- and sagittal-plane contours of the lumbar spine. Cylindrical and trapezoidal fusion cages and allograft femoral rings improve lumbar lordosis. Standalone interbody devices have also been used in cases of low-grade spondylolisthesis. Frontal-plane angular and translational deformities can be improved by implanting interbody fusion devices.

Immediate postoperative improvements in frontal- and sagittal-plane contours are not maintained over time in all cases. ${ }^{3,5,11}$ Stand-alone implants are susceptible to subsidence into the vertebral endplates. Subsidence of the implants, which occurs over the course of several years postoperatively, often leads to segmental spinal instability, loss of lordosis, angular frontal-plane deformities, and sagittal-plane translation. Subsidence is evidence of a delayed fusion or frank pseudarthrosis (Fig. 2). The ability of an implant to resist subsidence is, in part, related to its design (unpublished data, Burkus and colleagues). Subsidence, loss of disc space height, and angular deformity are also related to the position of the implants within the disc space. ${ }^{3}$

Interbody fusion can only be determined to be complete if no change in the alignment of the spine at the instrumented site is demonstrated for a minimum of 6 months postoperatively. Standing AP and lateral x-ray films must reveal no significant change in segmental lordosis, sagittal translation, or frontal-plane angulation on sequential radiographs acquired at least 6 months apart. For example, there can be evidence of angular change between radiographs obtained immediately and 6 months after surgery, but no change between 6 months and 1 year should be evi- denced. Fusion criteria would be partially met at 1 year postimplantation. Interbody fusion cannot be considered intact if there are progressive changes in any frontal- or sagittal-plane angular or translational measurements (Fig. $3)$. Standing x-ray films should be assessed for these changes because $\mathrm{x}$-ray films obtained with the patient

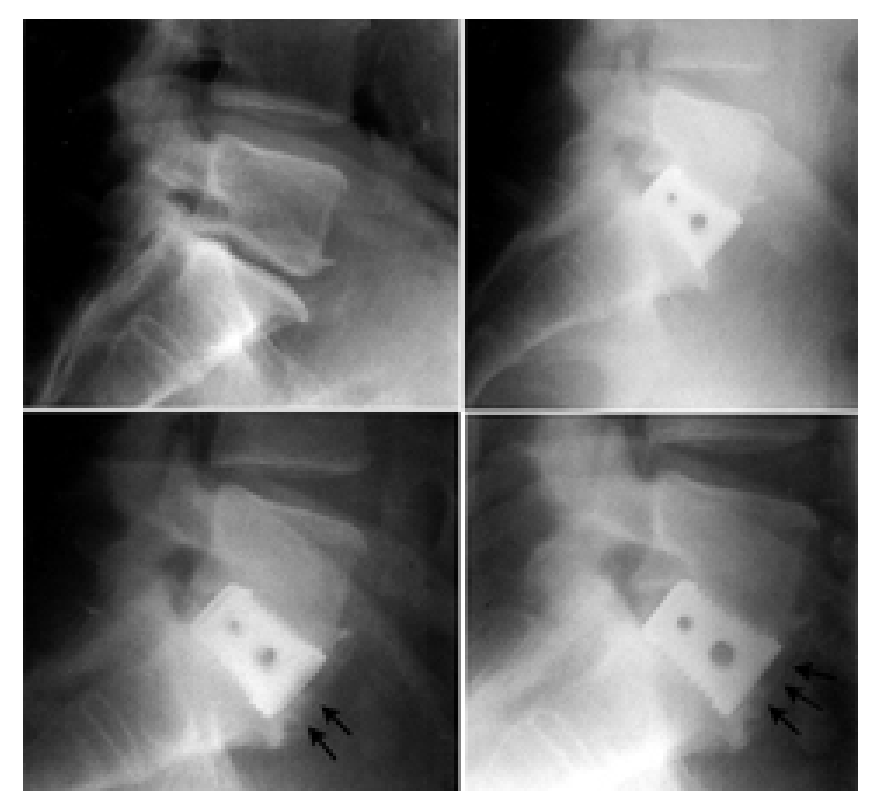

Fig. 3. Upper Left: Lateral radiograph revealing severe disc space collapse (posterior slippage) and radial bone spur formation at L5-S1. Upper Right: Radiograph obtained 6 weeks postoperatively deomonstrating an LT-Cage in place. There is restoration of the anatomical disc space height and restoration of the normal sagittal-plane contours. Lower Left: Lateral radiograph obtained 1 year postoperatively demonstrating no evidence of implant subsidence or change in sagittal-plane contours. Arrows point out new bone formation anterior to the cage. Lower Right: Lateral radiograph revealing no change from the 6-week or 1-year postoperative radiographs. Bone has spontaneously formed anterior to the implants-a positive sentinel sign (arrows). 


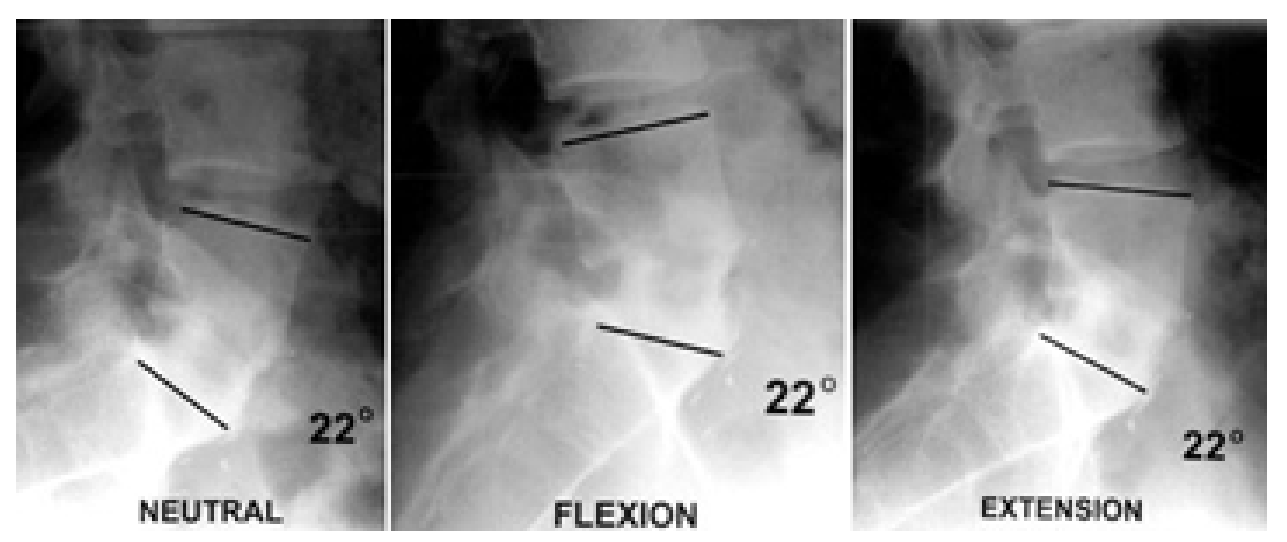

Fig. 4. Left: Standing lateral radiograph revealing incorporation of two threaded cortical allografts at the L5-S1 interspace. Center: Sitting lateral flexion radiograph demonstrating no change in lordosis at the L5-S1 interspace. There is significant angular change at the L4-5 interspace. Right: Sitting hyperextension lateral radiograph demonstrating no change in lordosis at the L5-S1 interspace and motion at the adjacent L4-5 interspace.

supine may not reveal findings associated with segmental instability.

\section{Dynamic Motion Studies}

Subtle changes in the lumbar contours can be identified on dynamic lateral radiographs; changes in segmental lordosis and sagittal-plane translation can be identified on these studies. In clinical studies, examiners have considered spinal motion segments to be fused despite measured differences in both angular and sagittal translation. ${ }^{7,12}$ Commonly, the presence of 3 to $5^{\circ}$ of motion at an instrumented segment and $5 \mathrm{~mm}$ of translation is considered to indicate successful fusion. These differences on dynamic studies are accepted because of measurement errors. Clinicians often find these results difficult to interpret because motion should not occur within a fused spinal segment. A standard method for obtaining, measuring, or interpreting the results of dynamic lumbar radiographic studies has not been established. Despite this absence, flexion-extension studies are widely reported in the literature.

Although biplanar studies are one of the most reliable plain radiographic modalities, there is no standard method for obtaining flexion-extension radiographs. Dynamic radiographs acquired with the patient in the standing position are not reliable because the technician cannot always properly center the x-ray beam at the appropriate interspace. In standing lateral dynamic studies, the pelvis is not locked and motion may be occurring at the hip joints rather than within the lumbar spine. Supine lateral dynamic studies obtained in the decubitus position are also somewhat unreliable for the same reasons-the pelvis is not locked or properly supported. Lateral x-ray films are frequently rotated unless the lumbar spine has been supported. It is technically difficult to maintain the x-ray beam parallel to the spinal motion segment during these dynamic studies. Flexion-extension radiographs should be obtained with the pelvis fixed so that motion occurs within the lumbar spine.

We recommend obtaining dynamic studies with the patient in the seated position. This position restricts pelvic and hip motion, enabling the technician to obtain radiographs consistently centered at the appropriate disc space with minimal rotational distortion at the interspace. Restricting motion to the lumbar spine by eliminating hip and pelvic motion and consistently obtaining radiographs that are parallel to the endplate of the instrumented segment sufficiently improves the accuracy of these dynamic studies; further, subtle changes within the spinal motion segment are demonstrated (Fig. 4). The accuracy of this method has not yet been quantified.

\section{Device-Bone Interface}

The reaction of host bone to an interbody fusion device helps determine fusion. Although the composition and shape of the implant significantly influence a variable region around the device, host bone reaction to the implant remains an important aspect of fusion. Plain radiographs, dynamic extension radiographs, and thin-cut CT scans are helpful in assessing the device-bone interface.

The presence of sclerosis surrounding an implant, the development of fibrous tissue reaction around an implant, and the migration and subsidence of the implant within the host bone are signs of instability, delayed union, and pseudarthrosis. Although these changes can be seen on plain radiographs, they are best depicted on thin-cut CT scans.

Plain radiographs can identify cyst formation within the subchondral bone, endplate sclerosis, and fibrous lucencies surrounding the implants. Additionally, they can identify migration (Fig. 5) and subsidence of the implants within the disc space. Cyst formation within the subchon-

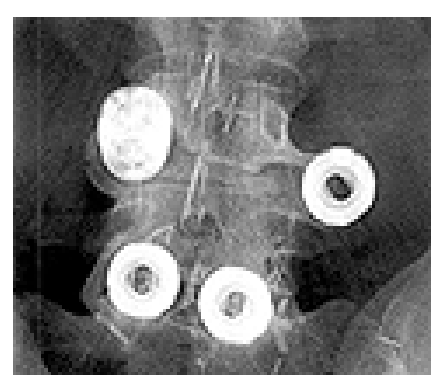

Fig. 5. Anteroposterior radiograph revealing lateral migration of a BAK cage at the L4-5 interspace. 


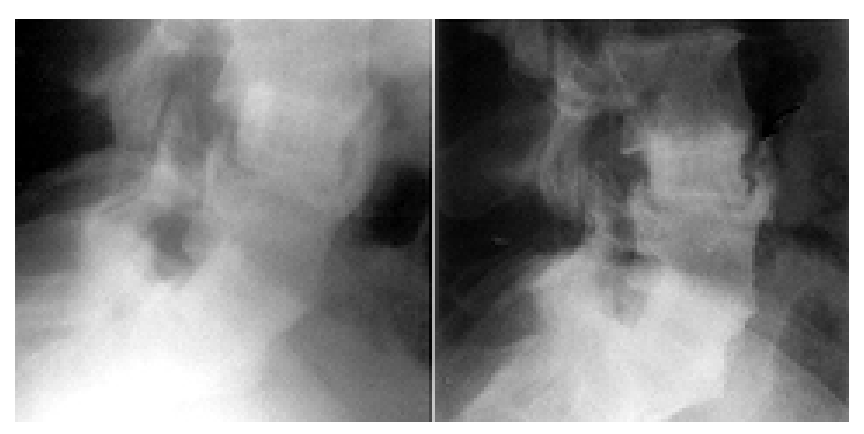

Fig. 6. Left: Standing lateral radiograph demonstrating threaded cortical dowels in place at the L4-5 and L5-S1 interspaces. There appears to be trabeculated bridging bone crossing both interspaces. Right: Sitting hyperextension lateral radiograph evidencing no change at the L5-S1 level. At the L4-5 level a gap appears between the implants and the anterior sentinel bone graft (arrow). The anterior graft has not attached to the L-4 vertebral body. The arrow points out the pseudarthrosis.

dral bone and adjacent to the implants indicates that micromotion at the interspace has occurred. Endplate sclerosis that extends through the subchondral bone and alters the trabecular pattern of the vertebral body is also consistent with micromotion and pseudarthrosis. The presence of radiolucencies surrounding the implants represents the interposition of fibrous tissue at the host bone-implant interface. It is commonly agreed that this is also a sign of micromotion and instability. Progressive collapse of the interspace and migration of implants are gross radiographic signs of instability.

Dynamic lateral extension radiographs help to identify subtle patterns of motion at the disc space as well as to help to identify the presence of interface lucencies. When hyperextension lateral radiography is performed the gap between the implant and host bone can be increased if fusion has not occurred. This gap appears as an increased radiolucency surrounding the implant or may appear as a gap in the anterior fusion mass (Fig. 6).

Thin-cut CT scans are also helpful in demonstrating cystic changes within the vertebral endplates, sclerosis, and interface lucencies. In one study the authors found CT scans of little value in assessing fusion for the first-generation BAK cages (unpublished data, Heithoff and coworkers). In cases in which the thick-walled and square-threaded BAK cage has been implanted it is difficult to assess for fusion because of the radiographic scatter inherently associated with the device's design. However, endplate sclerosis and the presence of cyst formation within the endplate adjacent to the implants can be readily determined even in patients who have received these first-generation implants (Fig. 7). The interface between the host bone and titanium implants can be more easily assessed after implantation of second-generation cages such as the INTER FIX threaded fusion cage (Medtronic Sofamor Danek, Memphis, TN) and LT-Cage (Medtronic Sofamor Danek). The thread patterns on these cages are self-tapping and thinner than those in earlier devices. The radiographic scatter and artifacts are reduced. With the secondgeneration cages, it is possible to assess the interface

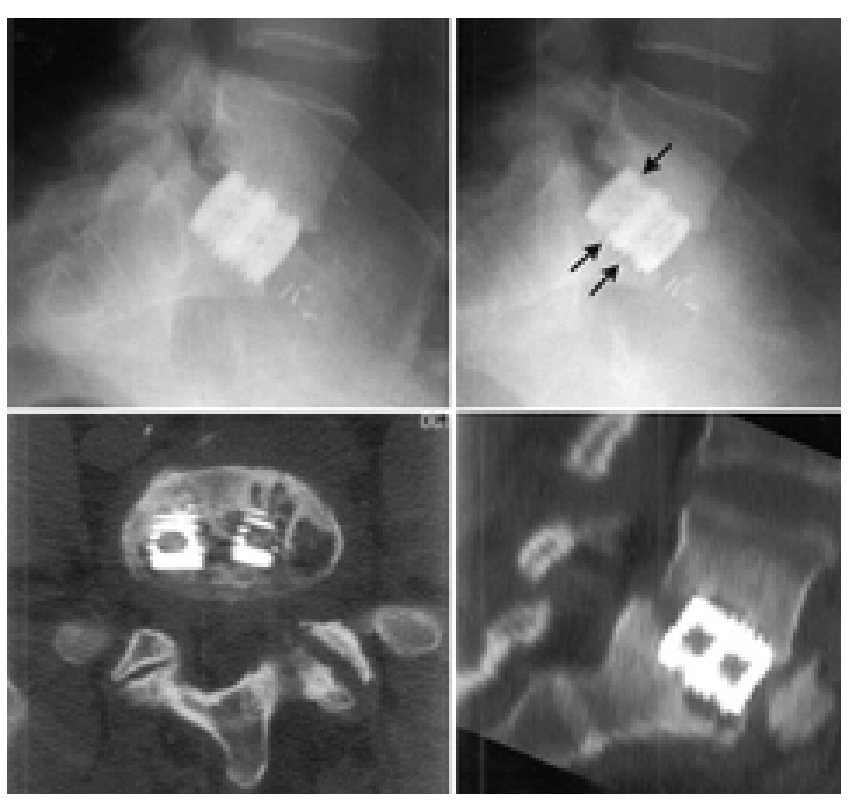

Fig. 7. Upper Left: Standing lateral radiograph obtained 4 weeks postoperatively demonstrating good position of two BAK cages in the L5-S1 interspace. Upper Right: Lateral radiograph obtained 1 year postoperatively revealing subsidence of the cages into the sacrum as well as radiolucencies around both cages. The arrows point to cystic changes within the adjacent vertebral endplates. Lower Left: Axial CT scan obtained across the L-5 endplate revealing lucencies surrounding both implants as well as cyst formation extending into the subchondral bone. Lower Right: Sagittal CT reconstruction depicting radiolucencies surrounding the BAK implant. A sentinel sign of anterior bone formation is present; however, this new bone formation extends well outside the disc space and is not attached to either vertebra. This is a sign of instability, not fusion.

between the implant and the host bone for the development of fibrous lucencies.

For stand-alone titanium interbody fusion constructs and threaded cortical allograft dowels, there are four points of contact that can be evaluated on both plain radiographs and CT scans (Fig. 8). These contact points involve the superior and inferior surfaces of each cage.

The assessment of interbody fusion when cortical allografts are used must include incorporation of the graft materials as well as the morselized autogenous grafts. Criteria for complete fusion of an allograft-autograft montage must include evidence of incorporation of the allograft into both vertebral endplates and trabecular bone formation across the interspace. ${ }^{17,25}$

In the assessment of threaded cylindrical bone dowels, incorporation of the allograft into endplates of the host vertebra can be determined. On plain radiographs and CT scans, it is common to find early trabecular bone formation crossing the interspace. In the presence of spanning trabecular bone formation around the implant, there is often incorporation of the allograft dowels to only one vertebral endplate. The lucencies surrounding the contact points of the allograft to one endplate often resolve over time (unpublished data, Burkus and associates). ${ }^{1}$ They are commonly present 1 year postoperatively and do not 


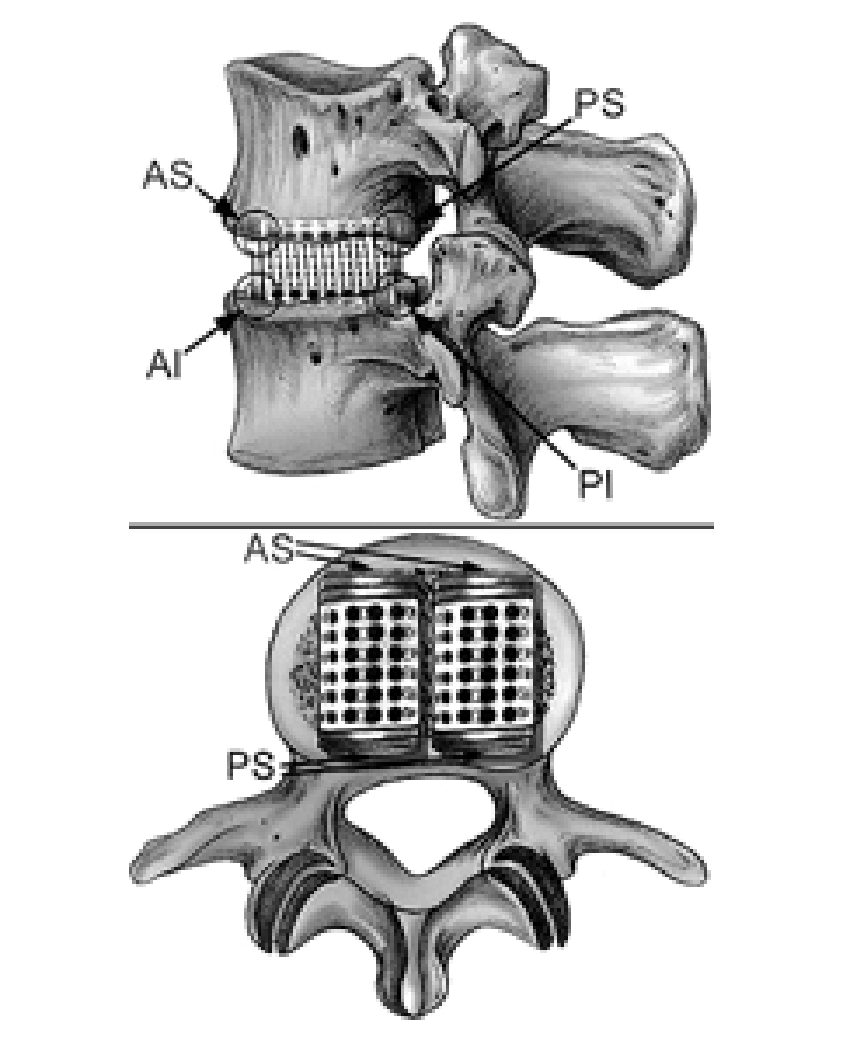

Fig. 8. Schematic drawings. Upper: A lumbar interspace with two interbody fusion cages in place. Four contact points between the implant and host bone are identified. Lower: Schematic representation of contact points demonstrated on an axial CT scan with two fusion cages placed at the superior endplate of a lumbar disc space. The four contact points are: $\mathrm{AS}=$ anterosuperior; $\mathrm{AI}=$ anteroinferior; $\mathrm{PS}=$ posterosuperior; $\mathrm{PI}=$ posteroinferior.

resolve until 2 to 3 years. These unilateral lucencies demonstrated on dynamic plain radiographs are not associated with poor clinical outcomes, subsidence, or instability. They do represent, however, incomplete incorporation and fusion of the allograft.

\section{New Bone Formation and Bone Remodeling}

The presence of new bone formation and bone remodeling in and around interbody fusion cages can be assessed radiographically. Carbon fiber implants and cortical allografts are readily assessed radiographically. The ability to assess bone formation around titanium implants depends, in part, on the implant's size, its configuration, and its porosity. The first-generation BAK cage is thick walled and square threaded; it also has two small openings that are bordered by an internal strut for driving the implant. The configuration of this thick-walled titanium implant is not conducive to radiographic visualization of bone graft within the cage or immediately adjacent to it. Second-generation titanium implants produce less scatter and artifact on plain radiographs and CT scans. The INTER FIX, the INTER FIX RP (Medtronic Sofamor Danek), and Ray TFC (Surgical Dynamics, Inc., Norwalk, CT) ${ }^{16}$ fusion cages are hollow, fenestrated cylinders with no internal driving device. These cages are significantly more porous, and their thread patterns are also not square.

\section{Fusion Zones}

We have defined five zones of fusion that can be established for interbody devices. Identifying bone formation within the various zones is significant because different patterns of bone formation occur at different time intervals. Bone formation in different zones also indicates different prognostic significance in the determination of fusion. Trabecular bone formation within the fusion devices and anterior to the devices represents incorporation and maturation of the autogenous grafts. This pattern is difficult to discern accurately. New bone formation in a region or zone of the interspace that is free of autogenous graft or growth factors represents osteoinduction within the soft-tissue elements of the spinal motion segment. Identification of osteoinduction within the disc space is the most accurate means of determining fusion after an ALIF procedure, as new bone formation only occurs in a spinal motion segment that is adequately stabilized and, therefore, represents a fused motion segment. The zones of fusion can be assessed using plain radiography and CT scanning.

Anterior Zone. The Anterior Zone is an area of bone formation in front of the cages along the anterior margins of the disc space (Fig. 9). Bone formation within this zone is probably the least reliable indication that interbody fusion has occurred. The formation of radial osteophytes, which is indicative of instability, often masquerades as an early sentinel sign. On the basis of anterior bone formation alone, it is impossible to discern if it is a good sign or a bad sign. For fusion to be present, trabecular bone formation in the Anterior Zone must be complete from endplate to endplate. Bone formation that extends past the confines of the disc space can be an early indication of developing pseudarthrosis.

Posterior Zone. The Posterior Zone is the posterior margin of the interspace (Fig. 9). Trabecular bone formation in this zone is most likely the best radiographic indication that interbody fusion has been achieved. In our clinical experience with stand-alone anterior fusion devices, bone formation in the Posterior Zone is the most reliable indication of fusion (Fig. 10).

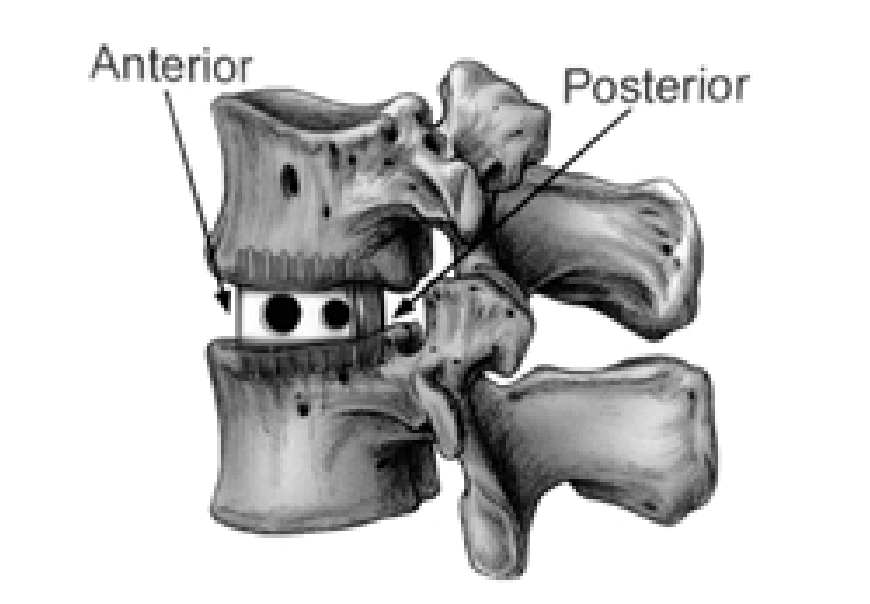

Fig. 9. Schematic drawing depicting the location of the Anterior and Posterior Fusion Zones. 


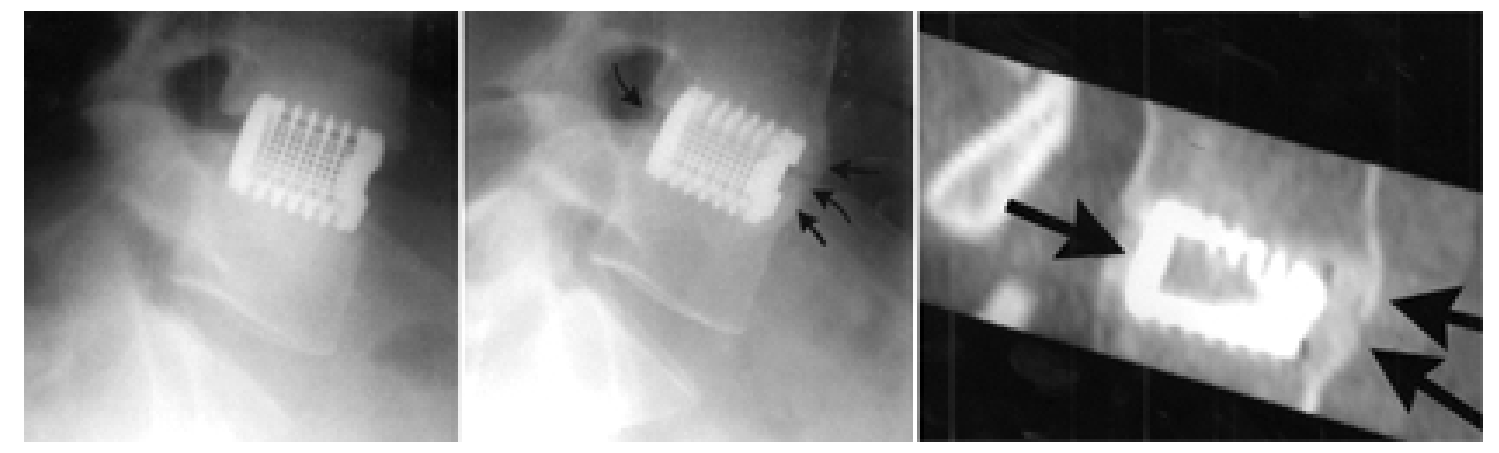

Fig. 10. Left: Lateral radiograph obtained 6 weeks after implantation of the INTER FIX cages in the L4-5 interspace. No bone is present in the Anterior or Posterior Fusion Zones. Center: Lateral radiograph obtained 1 year postoperatively revealing new bone formation in the Anterior and Posterior Fusion Zone. The single arrow points to trabecular bone formation behind the interbody fusion cage in the Posterior Fusion Zone. The three arrows point to spanning trabecular bone across the disc anterior to the implants. Right: Sagittal CT reconstruction demonstrating trabeculated bone present in the Anterior and Posterior Fusion Zones and connecting the adjacent vertebral endplates. The single arrow shows bone in the Posterior Fusion Zone. The dual arrows show bone in the Anterior Fusion Zone.

Lateral Zone. The Lateral Zone, or the lateral margins of the disc space, is divided into left and right regions (Fig. 11). Bone formation between the lateral borders of the implants and the annulus is difficult to visualize on plain radiographs. Ferguson-view radiographs of the L4-5 and L5-S1 interspace rarely demonstrate early bone formation in the Lateral Zone. Computerized tomography scanning is essential in visualizing early trabecular bone formation. Only the final stages of ossification of the annulus fibrosus are apparent on plain AP radiographs. Early bone formation between the annulus and implant can be readily visualized on CT scans (Fig. 12). The presence of bone formation in these zones is also a very good predictor of fusion and typically occurs here before it does in the Posterior Zone. Bone formation is often asymmetrical and may be related to cage placement. It often influences the progression of the trabecular bone growth patterns within the cages.

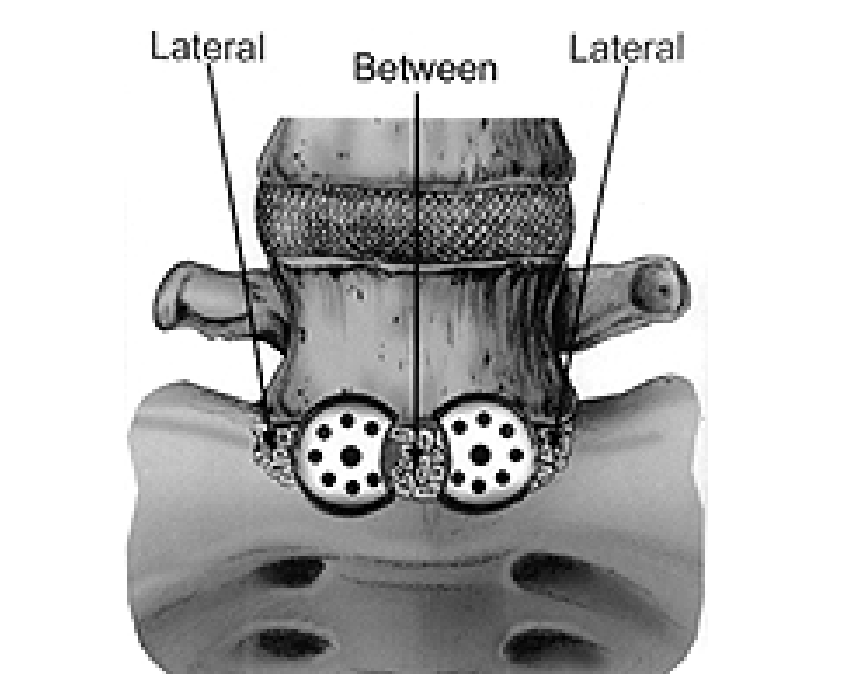

Fig. 11. Schematic drawing depicting the location of the Lateral and Between Fusion Zones.
Between Zone. The Between Zone is the area of bone formation between the implants (Fig. 11). Bone formation in this zone is best visualized on thin-cut CT scans. However, when two reduced lateral profile implants (that is, INTER FIX RP cages) are used adjacent to each other within a disc space, the large central opening between the implants allows for adequate visualization of bone formation between the cages even on plain AP radiographs (Fig. 13).

Within Zone. The Within Zone is the area of bone formation within the interbody fusion device (Fig. 14). This zone is often subdivided into two compartments: one for each device placed within the disc space. It is very difficult to differentiate between living and dead bone within the cages. The size, configuration, and material of the cages also significantly influence our ability to assess bone formation accurately in this zone (Fig. 15). Assessment of bone formation is not practical with a single CT scan. It is best assessed over time by obtaining serial scans. A significant error rate is associated with the assessment of increasing density within the cages. ${ }^{1}$ Scanning performed in patients with known phantom bone density calibration devices can diminish the error.

Peripheral Zone. The Peripheral Zone comprises the posterior facet joints of the fused spinal motion segment.

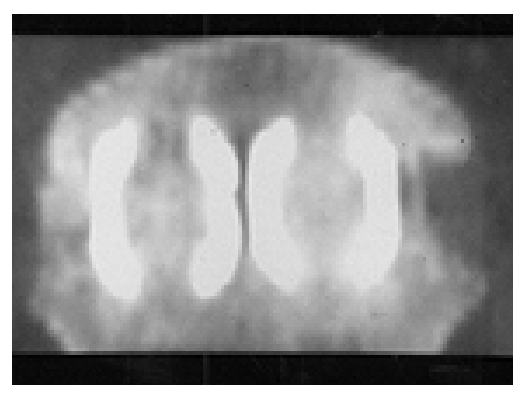

Fig. 12. Frontal-plane CT recontruction revealing bone formation lateral to implants within disc space (Lateral Fusion Zones). 


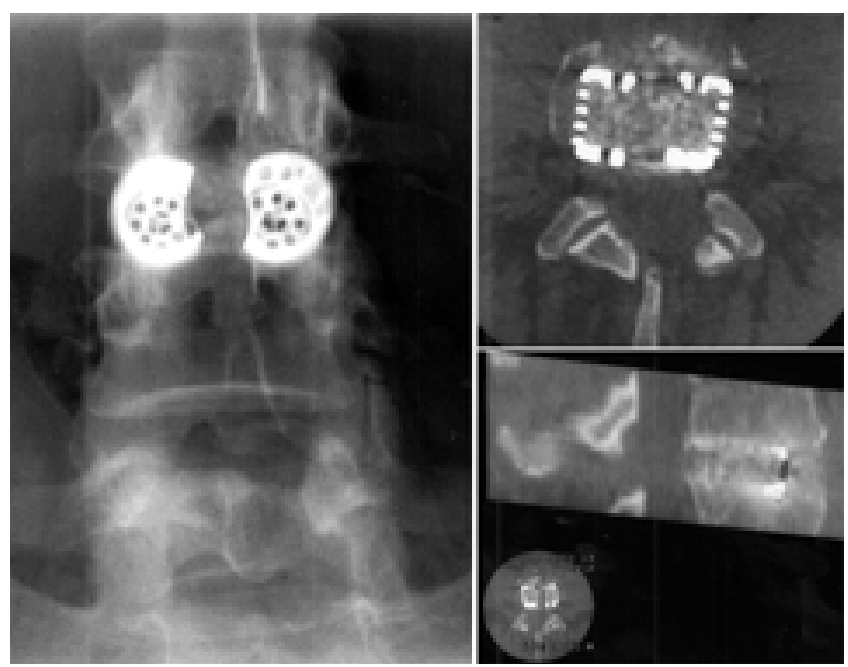

Fig. 13. Left: Ferguson-view radiograph of the L3-4 interspace revealing two INTER FIX RP fusion cages placed adjacent to each other. Trabeculated bone spanning the disc space is seen between the implants (Between Fusion Zone). Upper Right: Axial CT scan obtained through the central portion of the disc space, demonstrating trabecular bone formation. Lower Right: Sagittal CT reconstruction obtained through the central portion of the disc space, demonstrating trabecular bone formation crossing the disc space.

After a successful interbody fusion, the posterior facet joints become ankylosed over time. Facet joint fusion can best be vizualized on thin-cut CT scans.

\section{CONCLUSIONS}

Radiographic criteria have been established to assess fusion reliably following ALIF. These criteria are useful in determining whether threaded and impacted implants and titanium, carbon fiber, and allograft devices have successfully achieved fusion. Determination of fusion involves the radiographic evaluation of spinal alignment, angular and translational changes viewed on dynamic motion studies, assessment of the device-host interface, and identification of new bone formation and bone remodeling.

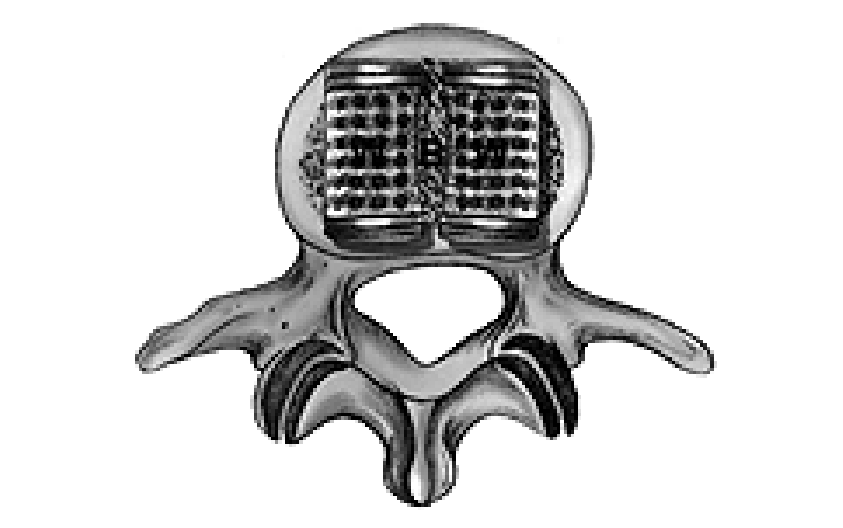

Fig. 14. Schematic drawing depicting the location of the Within Fusion Zone (W) and Between Fusion Zone (B).

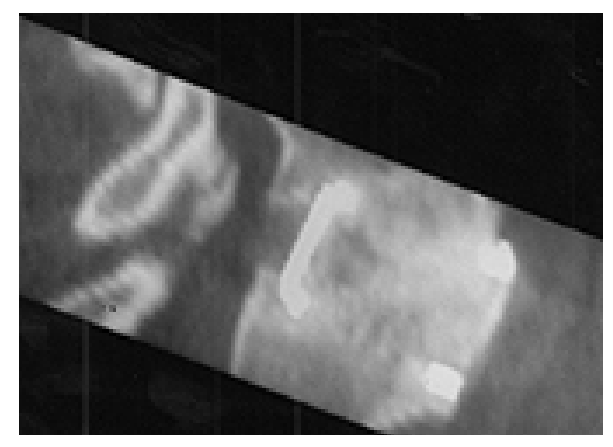

Fig. 15. Sagittal CT reconstruction through the midportion of an LT-Cage at 1 year postoperatively. The thin-walled and porous LT-Cage produces minimal radiographic scatter and artifact. Uninterrupted trabecular bone growth can be seen within the cage (Within Fusion Zone) spanning the disc space.

Each of these criteria must be met to ensure that fusion is complete within a vertebral motion segment.

Spinal alignment is assessed on plain radiographs obtained with the patient in a standing position. Evaluation should be conducted sequentially over a minimum of 6 months. Any radiographically demonstrated changes within the spinal motion segment over time indicate a delayed fusion. Progressive subsidence or any change in sagittal- or frontal-plane contours represents a failure of fusion. A fusion can be considered intact if there is no change in spinal alignment within the instrumented motion segment over a 6-month period (acceptable intraobserver measurement error is $3^{\circ}$ ).

Dynamic radiographic studies must be obtained in a manner that applies stress to the instrumented spinal motion segment. The pelvis must be stabilized and radiographs must be obtained parallel to the endplates of the fused segment. Changes in sagittal-plane contours or the appearance of lucencies at the implant-endplate interface are indicative of a failure of fusion. If pseudarthrosis is present, a hyperextension lateral radiograph frequently reveals a gap within an anterior sentinel fusion. Fusion is considered intact only if there is no significant motion revealed on dynamic studies (acceptable intraobserver measurement error is $3^{\circ}$ or $3 \mathrm{~mm}$ of translation).

The host bone reaction to the intradiscal implant must be assessed on plain radiographs and CT scans. Secondgeneration cages permit a closer evaluation of the host bone-implant interface. The development of cystic or sclerotic changes within the subchondral bone of the vertebral endplates is suggestive of a fusion failure. Incorporation of allograft bone into the vertebral endplates clearly indicates a solid arthrodesis. The absence of progressive radiographic lucencies around the metal of a carbon fiber implant is consistent with fusion.

Finally, the formation of new bone adjacent to or within the intradiscal implants is the most reliable finding indicating fusion. New bone formation occurs outside of the intradiscal implants when a fusion has occurred. New bone formation within the Lateral and Posterior Zones is the most reliable radiographic indication of a fusion. Remodeling of autogenous grafts or allografts is also consistent with an intact fusion. 


\section{Acknowledgments}

The concepts and principles discussed in this manuscript are the result of the collaborative efforts of the Surgical Interbody Research Group whose membership includes the following physicians: C. Branch, M.D.; J. K. Burkus, M.D.; R. Haid, M.D.; K. Foley, M.D.; G. Frey, M.D.; M. Gornet, M.D.; S. Heim, M.D.; S. Kitchel, M.D.; Professor J. C. LeHuec; H. H. Mathews, M.D.; J. Peloza, M.D.; W. Rauschning, M.D.; G. Rodts, M.D.; H. Sandhu, M.D.; R. Sasso, M.D.; T. Schuler, M.D.; C. Shaffrey, M.D.; R. Watkins, M.D.; and T. Zdeblick, M.D. The members of this group use the standards described here in the evaluation of interbody fusion.

\section{References}

1. Cizek GR, Boyd LM: Imaging pitfalls of interbody spinal implants. Spine 25:2633-2636, 2000

2. Crock HV: Anterior lumbar interbody fusion: indications for its use and notes on surgical technique. Clin Orthop 165: 157-163, 1982

3. Dennis S, Watkins R, Landaker S, et al: Comparison of disc space heights after anterior lumbar interbody fusion. Spine 14: 876-878, 1989

4. Djukic S, Lang P, Morris J, et al: The postoperative spine. Magnetic resonance imaging. Orthop Clin North Am 21:603-624, 1990

5. Dorchak JD, Burkus JK, Foor BD, et al: Dual paired proximity and combined BAK/proximity interbody fusion cages: radiographic results, in Proceedings of the North American Spine Society. New Orleans: North American Spine Society, 2000, pp 83-85 (Abstract)

6. Flynn JC, Hoque MA: Anterior fusion of the lumbar spine. Endresult study with long-term follow-up. J Bone Joint Surg Am 61:1143-1150, 1979

7. Frymoyer JW, Hanley EN Jr, Howe J, et al: A comparison of radiographic findings in fusion and nonfusion patients ten or more years following lumbar disc surgery. Spine 4:435-440, 1979

8. Hannon KM, Wetta WJ: Failure of technetium bone scanning to detect pseudarthroses in spinal fusion for scoliosis. Clin Orthop 123:42-44, 1977

9. Harmon PH: Anterior excision and vertebral body fusion operation for intervertebral disk syndromes of the lower lumbar spine: three- to five-year results in 244 cases. Clin Orthop 26: 107-127, 1963

10. Kant AP, Daum WJ, Dean SM, et al: Evaluation of lumbar spine fusion. Plain radiographs versus direct surgical exploration and observation. Spine 20:2313-2317, 1995
11. Kumar A, Kozak JA, Doherty BJ, et al: Interspace distraction and graft subsidence after anterior lumbar fusion with femoral strut allograft. Spine 18:2393-2400, 1993

12. Kuslich SD, Ulstrom CL, Griffith SL, et al: The Bagby and Kuslich method of lumbar interbody fusion. History, techniques, and 2-year follow-up of a United States prospective, multicenter trial. Spine 23:1267-1279, 1998

13. Lane JD Jr, Moore ES Jr: Transperitoneal approach to the intervertebral disc in the lumbar area. Ann Surg 127:537-551, 1948

14. McAfee PC: Interbody fusion cages in reconstructive operations on the spine. J Bone Joint Surg Am 81:859-880, 1999

15. Pearcy M, Burrough S: Assessment of bony union after interbody fusion of the lumbar spine using a biplanar radiographic technique. J Bone Joint Surg Br 64:228-232, 1982

16. Ray CD: Threaded fusion cages for lumbar interbody fusions. An economic comparison with 360 degrees fusions. Spine 22: 681-685, 1997

17. Rothman SL, Glenn WV: CT evaluation of interbody fusion. Clin Orthop 193:47-56, 1985

18. Sacks S: Anterior interbody fusion of the lumbar spine. Indications and results in 200 cases. Clin Orthop 44:163-170, 1966

19. Siambanes D, Mather S: Comparison of plain radiographs and CT scans in instrumented posterior lumbar interbody fusion. Orthopedics 21:165-167, 1998

20. Sørenson KH: Anterior interbody lumbar spine fusion for incapacitating disc degeneration and spondylolisthesis. Acta Orthop Scand 49:269-277, 1978

21. Stevenson JS, Bright RW, Dunston GL, et al: Technetium-99m phosphate bone imaging: a method for assessing bone graft healing. Radiology 110:391-394, 1974

22. Taylor TKF: Anterior interbody fusion in the management of disorders of the lumbar spine. J Bone Joint Surg Br 52:784, 1970 (Abstract)

23. Watkins R: Anterior lumbar interbody fusion surgical complications. Clin Orthop 284:47-53, 1992

24. Weiner BK, Fraser RD: Spine update: lumbar interbody cages. Spine 23:634-640, 1998

25. Wimmer C, Krismer M, Gluch H, et al: Autogenic versus allogenic bone grafts in anterior lumbar interbody fusion. Clin Orthop 360: 122-126, 1999

Manuscript received February 23, 2001

Accepted in final form March 20, 2001.

Address reprint requests to: J. Kenneth Burkus, M.D., The Hughston Clinic, PC, 6262 Veterans Parkway, Columbus, Georgia 31908-9517. email: jkb66@knology.net. 